\title{
Vómitos en pediatría: Presentación de caso clínico y abordaje diagnóstico
}

\author{
Vomiting in pediatrics: Clinical case presentation and Diagnostic Approach
}

Luis Enrique Jovel Banegas*, Wilmer Alejandro Madrid Mejía**, José María Paz ***, Essy Lucia Umanzor****

\section{RESUMEN}

El vómito es uno de los síntomas que se presenta con mayor frecuencia en los centros de atención pediátrica. Sus causas son múltiples y abarcan cualquier sistema de nuestro organismo.

La estenosis del píloro es una condición común en la infancia con una incidencia de 2 a 5 casos por cada mil nacidos vivos. Esta suele presentarse entre la 3era semana y 3 meses de vida, siendo excepcional su debut en edad más tardía.

Se presenta el caso de femenina de 2 años de edad, con cuadro de vómitos de larga evolución como signo principal, con distintos tratamientos pero sin mejoría y mediante un estudio contrastado de vía digestiva superior y confirmado mediante endoscopia se llegó al diagnóstico de una obstrucción de hipertrófica congénita de píloro.

Después de una breve revisión de la fisiopatología del vómito presentamos un abordaje diagnóstico que pueda ser de utilidad para identificar la causa y ofrecer un tratamiento oportuno en los pacientes pediátricos con cuadro de vómitos.

\section{PALABRAS CLAVE}

Estenosis hipertrófica de píloro, vómitos, diagnóstico.

\section{ABSTRACT}

Vomiting is a symptom that occurs most

\footnotetext{
* Gastroenterólogo pediatra, HNMCR

** Residente de 3er año, Posgrado de Pediatría, Universidad Nacional Autónoma de Honduras

***Pediatra, Instituto Hondureño de Seguridad Social

****Médico general

Dirigir correspondencia a: a_wilmer@hotmail.com

Recibido: 15 de enero 2016 Aprobado: 19 de noviembre 2016
}

frequently in child care centers. There are many causes it involves many systems of our body.

Pyloric stenosis is a common condition in children with an incidence of 2 to 5 cases per thousand live births. This usually occurs between the 3 rd week and 3 months of life. It is exceptional its debut in later age.

A 2 years old female presented vomiting as the principal sign for a very long period of time. She had different treatments without improvement. Through a contrasted study of the superior digestive tract and an endoscopy, the diagnosis of obstruction congenital hypertrophic pyloric was made.

After a brief review of the pathophysiology of emesis and a diagnostic approach is presented. These can be helpful to identify the cause of emesis and provide timely therapy in vomiting children.

\section{KEYWORDS}

Hypertrophic pyloric stenosis, vomiting, diagnosis.

\section{INTRODUCCIÓN}

Los vómitos son un motivo frecuente de consulta en pediatría, sobre todo en urgencias y consultas de gastroenterología. El acto del vómito obedece a un mecanismo motor complejo que incluye el descenso del diafragma y contracciones intensas de la musculatura abdominal que conllevan a la elevación de la presión intragástrica y la eyección del contenido gastrointestinal a través de la boca. ${ }^{(1)}$ El vómito es un proceso en el cual se involucran diversos sistemas que tienen influencia sobre el centro del vómito. Este se localiza en la formación reticular del tronco del encéfalo y tiene 
principalmente receptores muscarínicos, de histamina, neuroquinina y serotonina. ${ }^{(2)}$

Ante las diversas patologías que pueden desencadenar el vómito, debemos de realizar una evaluación apropiada para dar una pronta solución, sin incurrir en exámenes innecesarios y sobre todo evitar complicaciones, que van desde cuadro agudos graves como la deshidratación a procesos crónicos de una alta morbilidad como la detención del crecimiento. A continuación, presentamos el caso de una paciente con vómitos de una larga evolución y posteriormente, el abordaje diagnóstico de los vómitos.

\section{REPORTE DE CASO}

Paciente femenina de 2 años de edad sin antecedentes patológicos de importancia, con historia desde el mes de nacimiento de presentar vómitos de contenido alimentario, 20 a 30 minutos después de alimentarla con leche materna. Al inicio recibió manejo como una enfermedad por reflujo gastroesofágico con procinéticos e inhibidores de la bomba de protones; y aunque la ganancia de peso era adecuada en los primeros 6 meses, llamaba la atención de la madre que comía en poca cantidad, aunque de forma frecuente, no presentaba distensión abdominal y su patrón defecatorio era normal; y nunca mejoro de los vómitos. Al iniciar la ablactación madre nota que el contenido del vómito no solo es leche, sino los fragmentos de comida parcialmente digeridos y que persisten tanto los vómitos como la alimentación escasa, pero en forma frecuente, cada dos o tres horas. La madre debido a que no mejoraba decide dar todos los alimentos en forma licuada y fraccionada con duración de hasta 1 hora en cada toma ya que de esta forma la niña no presentaba vómitos y la ganancia de peso era adecuada. Pero a los 18 meses la ganancia de peso ya no es adecuada, por lo que la madre administra un estimulante del apetito. La niña aumentó su ingesta de comida pero de nuevo presentó vómitos, por lo que nuevamente inicia con licuados en poca cantidad pero con frecuencia y duración aumentada pero con pobre ganancia peso; además, se agrega un nuevo síntoma que es distensión a nivel epigástrico, por lo que decide asistir a consulta. Al examen físico se encuentra una niña alegre, cooperadora, con talla adecuada para la edad, pero con peso para la talla en el score Z -2 y -3. Con buen estado de hidratación, sin compromiso cardiovascular, el abdomen distendido a nivel de epigastrio y cuadrante superior derecho sin onda peristáltica, sin dolor y depresible a la palpación, sin presencia de masas; resto del examen físico normal.

Los electrolitos séricos, las pruebas de función renal, los tiempos de coagulación y un examen general de orina normal. La biometría hemática con anemia leve de tipo microcítica e hipocrómica. Ante la semiología de los vómitos y la distensión gástrica se solicita serie esófago gastroduodenal para descartar alguna causa anatómica, la cual reportó una obstrucción del vaciado gástrico, con estómago dilatado e hipotónico y ausencia de paso del contraste al duodeno tras 1 hora del estudio.

(Ver figura No. 1 y 2).

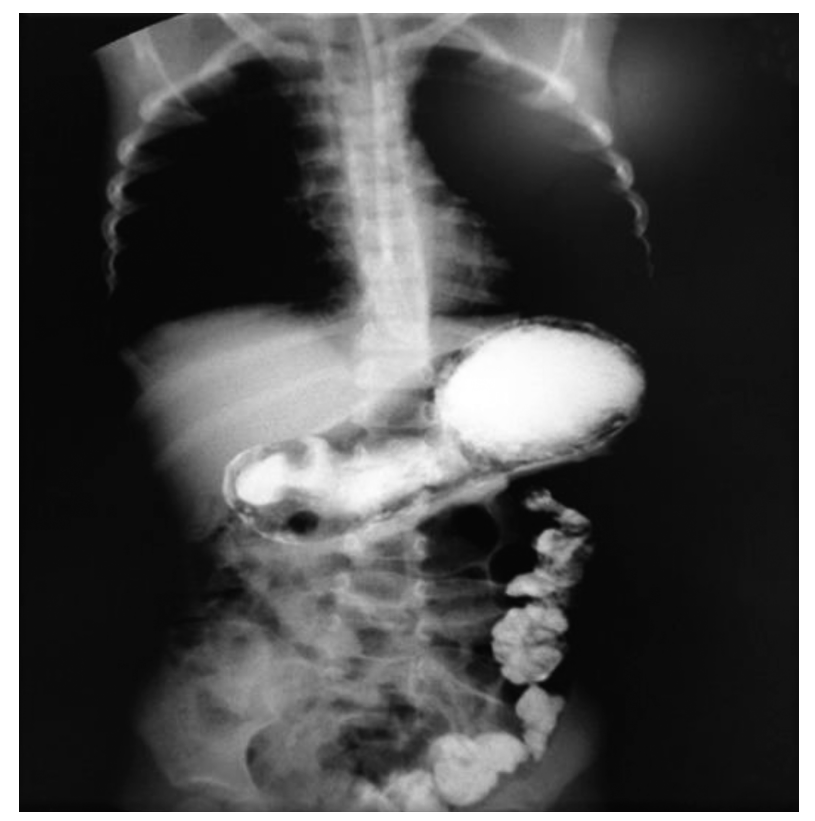

Figura No. 1: Se observa dilatación de la cámara gástrica y retardo de la salida de contraste. El colon descendente con medio de contraste remanente por Serie Esófago-GastroDuodenal del día previo a este estudio. 


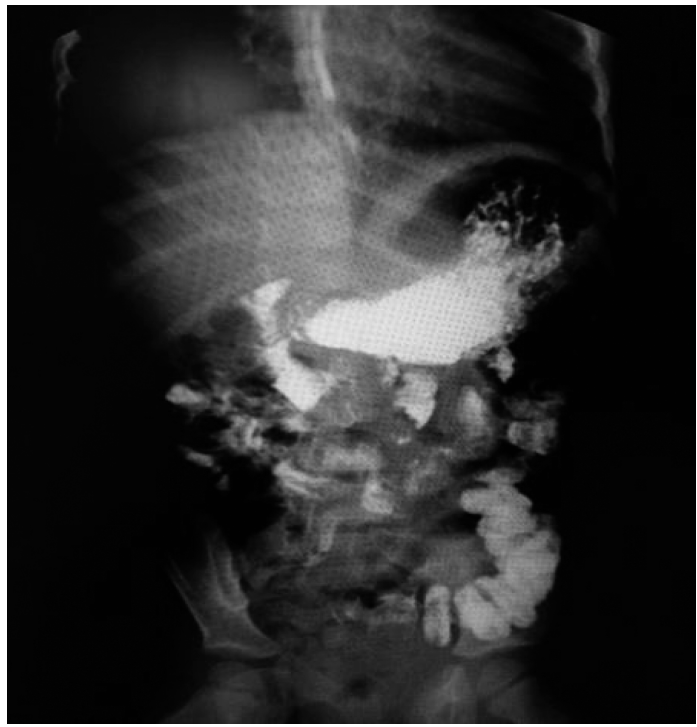

Figura No. 2: Muestra 2 horas después del uso de contraste: pequeña salida de material hacia el duodeno y al parecer, una obstrucción del tracto de salida a nivel del píloro.

Este estudio no fue concluyente para radiología, por lo que se realizó una endoscopía alta con el fin de ampliar estudios y valorar la anatomía interna del tracto intestinal superior, en la cual se pudo constatar una membrana fenestrada a nivel del tracto de salida (píloro) con 2 pequeños orificios por los cuales no se podía introducir el endoscopio neonatal. (Ver figura No. 3).

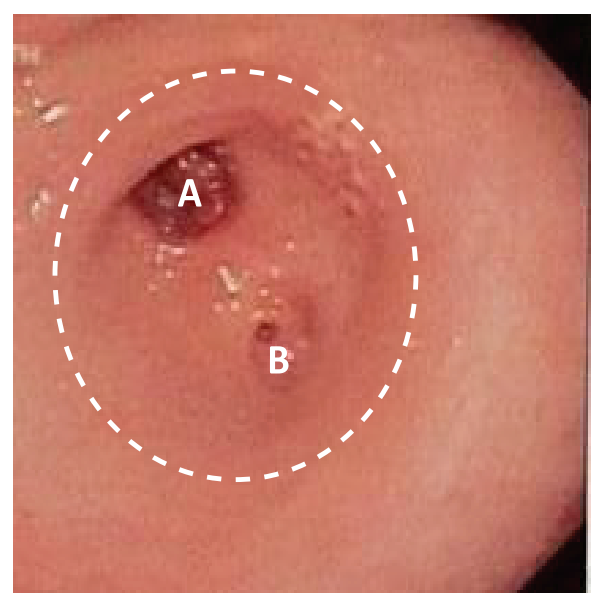

Figura No. 3: Endoscopía muestra el tracto de salida del tracto gástrico hacia el duodeno parcialmente cubierto por una membrana fenestrada con dos orificios (A y B) Al fondo del orificio $A$ se observa el pilórico.
Se realizó laparotomía exploradora encontrando un píloro engrosado, no se realizó piloromiotomía por el grosor del píloro sino una gastroduodenostomía para su resolución. El procedimiento se llevó a cabo sin complicaciones y se determinó como diagnóstico postoperatorio una estenosis hipertrófica del píloro.

Luego de la cirugía la paciente evolucionó satisfactoriamente, cumplió ayuno por 3 días, iniciando la transición a la vía oral con líquidos claros y luego con formula polimérica con buena tolerancia. Se egresó a los cuatro días con dieta para su edad, sin vómitos o distensión abdominal después de la ingesta de sus alimentos y en las consultas control continua sin vómitos y la ganancia de peso es adecuada.

\section{DISCUSIÓN}

El vómito consiste en la expulsión violenta por la boca del contenido del estómago y las porciones altas del duodeno provocado por un aumento de la motilidad gastrointestinal y contracciones involuntarias de la pared torácica y abdominal. ${ }^{(1)}$ Debe diferenciarse de la regurgitación en la cual hay un retorno del contenido gastrointestinal hacia la boca sin participación de la musculatura abdominal y el diafragma. El vómito es un síntoma con una variedad de diagnósticos diferenciales que varían desde lesiones propias del tracto gastrointestinal hasta enfermedades sistémicas. ${ }^{(1)}$

La estenosis pilórica ocurre en 2 a 5 niños de cada 1.000 nacidos vivos. Ocurre con mucha más frecuencia en los hombres, con una razón hombre: mujer de 4:1-6:1; y la edad de presentación más frecuente de la primera semana de vida al quinto mes vida extrauterina. ${ }^{(3)}$ Hasta un tercio de los casos de estenosis pilórica se ven en los primogénitos. Los factores genéticos juegan un papel en la estenosis pilórica, siendo mayor el riesgo en los gemelos monocigotos que en los gemelos dicigotos. ${ }^{(4,5)}$ Las principales complicaciones agudas son la deshidratación los trastornos electrolíticos y la alcalosis metabólica, todos secundario a los vómitos persistentes, y cuyo tratamiento es quirúrgico con una mortalidad quirúrgica de $0.5 \%$. 
El motivo de presentar este caso es proponer un abordaje diagnóstico en los pacientes pediátricos que se presentan a la consulta con vómitos y ofrecer herramientas clínicas prácticas para determinar la etiología.

El primer paso es determinar el estado de hidratación e iniciar reanimación con líquidos intravenosos en caso de compromiso hemodinámico. Las soluciones isotónicas son de elección, a una dosis de $20 \mathrm{ml}$ por kilogramo de peso a pasar lo más rápido posible. Una vez estabilizado el paciente debemos determinar la causa del vómito. ${ }^{(7)}$

El segundo paso será determinar si la causa es origen gastrointestinal o si es una enfermedad sistémica en relación a la edad. (Tabla 1 y 2 ). Cuando el origen es gastrointestinal puede ser obstructivo o secundario a una lesión erosiva/inflamatoria de la mucosa. Las características de vómito y síntomas acompañantes nos pueden ayudar a determinar esta diferencia, ya que las patologías obstructivas son las que requieren una pronta intervención, y se presentan sobre todo en recién nacidos y lactantes; a medida que aumenta la edad, las causas gastrointestinales no obstructivas y las causas no gastrointestinales son las más frecuentes. El vómito de contenido alimentario, postprandial, con sensación de plenitud a pesar de baja ingesta nos sugiere un cuadro obstructivo desde esófago hasta primera porción de duodeno. Si el vómito es de característica bilioso, acompañado de distensión abdominal, alteración en patrón defecatorio, ausencia de ruidos intestinales, la obstrucción probablemente sea a nivel de intestino delgado. Ante la sospecha de cuadro obstructivo la radiografía simple de abdomen y los estudios contrastados del tubo digestivo nos ayudaran a confirmar el sitio de la obstrucción, en algunos casos de obstrucciones digestivas altas la endoscopia también es una herramienta de utilidad. ${ }^{(1,9)}$

Los vómitos crónicos de contenido alimentario acompañado de irritabilidad en lactantes y dolor en epigastrio en niños mayores, y si se acompaña de hematemesis o melena, nos sugiere una lesión erosiva a nivel de esófago, estómago o primeras porciones de duodeno, probable secundario a una enfermedad por reflujo gastroesofágico o enfermedad acido péptica. En esta situación la historia alimentaria y antecedentes de uso de medicamentos son clave para el diagnóstico; aunque la enfermedad por reflujo gastroesofágico y la enfermedad acido péptica requieren un abordaje individual que no se discute en este artículo. Una endoscopia sería el estudio diagnóstico adecuado en esta situación.

Tabla No. 1: Causas más frecuentes de vómitos en neonatos, lactantes y preescolares.

\begin{tabular}{|c|c|c|c|c|}
\hline \multirow{2}{*}{\multicolumn{2}{|c|}{ Origen }} & \multicolumn{3}{|c|}{ Edad } \\
\hline & & \multirow[b]{2}{*}{\begin{tabular}{|l}
\multicolumn{1}{|c|}{ Neonatos } \\
-Hipertrofia \\
congénita de \\
píloro \\
-Bridas \\
congénitas \\
-Malrotación \\
intestinal con \\
vólvulo de \\
intestino \\
medio \\
-Atresia \\
intestinal \\
-íleo meconial \\
-Páncreas \\
anular
\end{tabular}} & Lactantes & \multirow[b]{2}{*}{\begin{tabular}{|l} 
Preescolares \\
\\
-Malrotación \\
intestinal con \\
vólvulo de \\
intestino \\
-Adherencias \\
-Obstrucción \\
por Áscaris \\
- Invaginación \\
intestinal
\end{tabular}} \\
\hline \multirow[t]{2}{*}{ 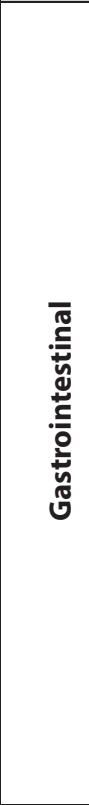 } & 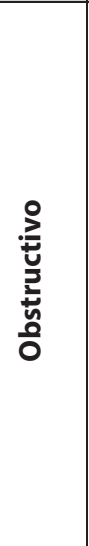 & & $\begin{array}{l}\text {-Hipertrofia } \\
\text { congénita de } \\
\text { píloro } \\
\text {-Invaginación } \\
\text { intestinal } \\
\text {-Malrotación } \\
\text { intestinal con } \\
\text { vólvulo de } \\
\text { intestino } \\
\text { medio }\end{array}$ & \\
\hline & 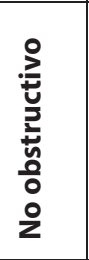 & $\begin{array}{l}\text {-APLV } \\
\text {-RGE o ERGE } \\
\text {-Enterocolitis } \\
\text { Necrotizante } \\
\text {-Enfermedad } \\
\text { de } \\
\text { Hirschsprung }\end{array}$ & $\begin{array}{l}\text {-APLV } \\
\text {-RGE o ERGE } \\
\text { Gastroenteri- } \\
\text { tis aguda }\end{array}$ & $\begin{array}{l}\text {-ERGE } \\
\text {-Gastroenteri- } \\
\text { tis aguda } \\
\text {-Esofagitis } \\
\text { eosinofílica } \\
\text {-Enfermedad } \\
\text { celiaca }\end{array}$ \\
\hline \multicolumn{2}{|c|}{$\begin{array}{l}\text { No } \\
\text { Gastrointestinal }\end{array}$} & $\begin{array}{l}\text {-ITU } \\
\text {-Infección de } \\
\text { SNC } \\
\text {-Enfermeda- } \\
\text { des metabó- } \\
\text { licas }\end{array}$ & $\begin{array}{l}\text {-Otitis media } \\
\text { aguda } \\
\text {-ITU } \\
\text {-Infección de } \\
\text { SNC } \\
\text {-Lesión } \\
\text { intracraneal } \\
\text {-Enfermedad- } \\
\text { es metabólicas } \\
\text {-Abuso infantil }\end{array}$ & $\begin{array}{l}\text {-ITU } \\
\text {-Faringitis } \\
\text { aguda } \\
\text {-Infección de } \\
\text { SNC } \\
\text {-Lesión } \\
\text { intracraneal } \\
\text {-Intoxicación/ } \\
\text {-envenenami- } \\
\text { ento } \\
\text {-Insuficiencia } \\
\text { adrenal }\end{array}$ \\
\hline
\end{tabular}

APLV: alergia a la proteína de la leche de vaca, RGE: Reflujo gastroesofágico, ERGE: enfermedad por reflujo gastroesofágico, ITU: infección del tracto urinario, SNC: sistema nervioso central Fuente: Parashette KR; Croffie J. Vomiting. Pediatrics in Review ${ }^{(1)}$ 
Tabla No. 2: Causas más frecuentes de vómitos en escolares y adolescentes.

\begin{tabular}{|c|c|c|c|}
\hline \multirow{2}{*}{\multicolumn{2}{|c|}{ Origen }} & \multicolumn{2}{|c|}{ Edad } \\
\hline & & Escolares & \begin{tabular}{|l|} 
Adolescentes \\
\end{tabular} \\
\hline \multirow{2}{*}{ 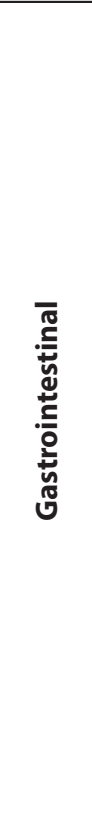 } & 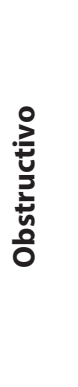 & $\begin{array}{l}\text { - Malrotación } \\
\text { intestinal con } \\
\text { vólvulo de intestino } \\
\text { medio } \\
\text { - Adherencias } \\
\text { - Obstrucción por } \\
\text { Áscaris } \\
\text { - Tumores } \\
\text { - Estenosis (Ell, } \\
\text { AINE's y radiación) }\end{array}$ & $\begin{array}{l}\text { - Malrotación } \\
\text { intestinal con } \\
\text { vólvulo de intestino } \\
\text { medio } \\
\text { - Adherencias } \\
\text { - Obstrucción por } \\
\text { Áscaris } \\
\text { - Tumores } \\
\text { - Estenosis (Ell, } \\
\text { AINE's y radiación) }\end{array}$ \\
\hline & 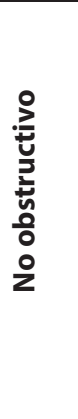 & $\begin{array}{l}\text { - Apendicitis aguda } \\
\text { - ERGE } \\
\text { - EAP } \\
\text { - Pancreatitis aguda } \\
\text { - Gastroenteritis } \\
\text { aguda } \\
\text { - Esofagitis } \\
\text { eosinofílica } \\
\text { - Enfermedad celiaca } \\
\text { - Ell }\end{array}$ & $\begin{array}{l}\text { - Apendicitis aguda } \\
\text {-ERGE } \\
\text { - EAP } \\
\text { - Pancreatitis aguda } \\
\text { - Gastroenteritis } \\
\text { aguda } \\
\text { - Esofagitis } \\
\text { eosinofílica } \\
\text { - Ell } \\
\text { - Síndrome de } \\
\text { vómitos cíclicos }\end{array}$ \\
\hline \multicolumn{2}{|c|}{$\begin{array}{l}\text { No } \\
\text { Gastrointestinal }\end{array}$} & $\begin{array}{l}\text { - ITU } \\
\text { - Infección de SNC } \\
\text { - Lesión intracraneal } \\
\text { - Intoxicación/ } \\
\text { - envenenamiento } \\
\text { - Insuficiencia adrenal }\end{array}$ & $\begin{array}{l}\text { - Otitis media aguda } \\
\text { - ITU } \\
\text { - Infección de SNC } \\
\text { - Lesión intracraneal } \\
\text { - Coledocolitiasis } \\
\text { - Diabetes } \\
\text { - Embarazo } \\
\text { - Abuso de drogas } \\
\text { - Migraña }\end{array}$ \\
\hline
\end{tabular}

Ell: Enfermedad inflamatoria intestinal, AINE's: antiinflamatorio no esteroideos, ERGE: enfermedad por reflujo gastroesofágico, EAP: enfermedad acido péptica, ITU: infección del tracto urinario, SNC: sistema nervioso central,

Fuente: Parashette KR; Croffie J. Vomiting. Pediatrics in Review ${ }^{(1)}$

La instauración aguda del vómito acompañado de dolor abdominal severo puede sugerir una patología de origen quirúrgico como invaginación intestinal en lactantes y apendicitis en escolares y adolescentes; o cuadros no quirúrgicos como pancreatitis aguda, el diagnóstico lo hacemos clínico, con apoyo de biometría hemática, enzimas pancreáticas y estudios de imagen (ultrasonido, tomografía contrastada de abdomen) que nos ayudan a confirmar el diagnóstico. Con el vómito de presentación aguda pero acompañado de diarrea y fiebre nos sugiere un proceso infeccioso agudo de etiología viral, bacteriana, o parasitaria, el diag- nóstico es clínico; pero cuando el vómito se acompaña de diarrea, dolor abdominal y fiebre, pero estos síntomas son persistentes o crónicos y asociados a detención del crecimiento (perdida o pobre ganancia de peso/talla) nos sugiere una enfermedad inflamatoria intestinal o enfermedad celiaca. Junto a la clínica, los estudios de laboratorio van dirigidos a la búsqueda de anticuerpos específicos; así como, estudios endoscópicos y biopsias.

Si no comprobamos una causa gastrointestinal, ya sea obstructiva o no obstructiva, debemos de sospechar causas extraintestinales, las cuales pueden ser de distintos órganos. El vómito en proyectil nos obliga a investigar una causa intracranena, por lo que la toma de la presión arterial y un examen neurológico exhaustivo incluyendo el fondo de ojo son obligatorios para descartar hipertensión endocraneana secundaria a múltiples patologías del sistema nervioso central; debemos solicitar tomografía cerebral, resonancia magnética y hasta punción lumbar como complemento diagnóstico. El vómito de presentación aguda acompañado de fiebre y ausencia de síntomas gastrointestinales acompañantes, nos deben sugerir una infección en otro sitio, desde vía aérea, tracto urinario y sistema nerviosos central. En esta circunstancia el diagnóstico es clínico y las pruebas complementarias deberán realizarse en base a los hallazgos en el examen físico, que van desde un examen general de orina, urocultivo, hemocultivo y punción lumbar.

El vómito que es recurrente o crónico acompañado de detención de peso y talla puede sugerir una patología renal ya sea de tipo anatómico o metabólico como la acidosis tubular renal, por lo que debemos solicitar examen de orina, urocultivo, ultrasonido renal, estudios contrastados de vía urinaria, creatinina, nitrógeno ureico, niveles de bicarbonato y electrolitos séricos en sangre y orina. Otras patologías que presentan vómitos crónicos o persistentes acompañado detención de crecimiento, síntomas de hipoglucemia, neurológicos o hepáticos son las enfermedades metabólicas de 
carbohidratos, lípidos o proteínas. El tamiz neonatal sería la prueba de elección en recién nacidos, pero el estudio de estas enfermedades es más complejo y se basa en la medición de la actividad enzimática en el órgano afectado o mediante estudio genético. Otros trastornos deben ser investigados, como los endocrinos (hipotiroidismo, diabetes mellitus).

El uso de medicamentos puede provocar vómito como parte de sus efectos adversos por ejemplo los antineoplásicos. Otros pueden provocar vómito debido a su ingesta excesiva como el hierro y digoxina. En niños mayores y adolescentes se debe preguntar sobre abusos de sustancias como alcohol, marihuana y trastornos psiquiátricos como bulimia o depresión.

La lista de causas de vómito es amplia, pero una buena historia clínica, que incluya la semióloga del vómito, síntomas acompañantes, historia alimentaria y de uso de medicamentos, la valoración del peso y la talla y un examen físico apropiado, nos va a orientar a determinar la causa y de esta forma solicitar los estudios complementarios adecuados, sin gastos innecesarios; y sobre todo realizar un diagnóstico oportuno.

\section{BIBLIOGRAFÍA}

1. Parashette KR; Croffie J. Vomiting. Pediatrics in Review 2013;34;307.

2. Quigley, EM; Hasler, WL; Parkman, HP. AGA Technical Review on Nausea and Vomiting. Gastroenterology 2001;120:263-286.

3. Kliegman RM; Behrman, RE. Estenosis Hipertrófica de Píloro Tratado de Pediatría de Nelson 18 Ed, cap. 326: 1555- 1557.

4. Boybeyi O, Karnak I, Ekinci S, Ciftci AO, Akçören Z, Tanyel FC. Late-onset hypertrophic pyloric stenosis: definition of diagnostic criteria and algorithm for the management. J Pediatr Surg. 2010 Sep;45(9):1777-83.

5. Honein MA, Paulozzi LJ, Himelright IM, et al. Infantile hypertrophic pyloric stenosis after pertussis prophylaxis with erythromycin: a case review and cohort study. Lancet. 1999;354(9196): 2101-2105.

6. Y. Martínez-Criado, D. Aspiazu, R. Cabrera, J.C. De Agustín. Delayed presentation of hypertrophic pyloric stenosis in infants: an unusual case, Elsevier Vol. 76. Núm. 03. March 2012.

7. Quigley EMM, Hasler WL, Parkman HP. AGA technical review on nausea and vomiting. Gastroenterology. 2001;120(1):263-286.

8. Furuta GT, Liacouras CA, Collins $\mathrm{MH}$, et al; First International Gastrointestinal Eosinophil Research Symposium (FIGERS) Subcommittees. Eosinophilic esophagitis in children and adults: a systematic review and consensus recommendations for diagnosis and treatment. Gastroenterology. 2007;133(4): 1342-1363.

9. Chial HJ, Camilleri M, Williams DE, Litzinger K, Perrault J. Rumination syndrome in children and adolescents: diagnosis, treatment, and prognosis. Pediatrics. 2003;111(1):158-162.

10. Gencosmanoglu R, Sad O, Sav A, Tozun N. Primary hyper- trophic pyloric stenosis in the adult: A case report. Turk J Gastroenterol 2002;13(3):175-9.

11. Hernanz-Schulman M. Infantile hypertrophic pyloric stenosis. Radiology 2003;227:219-331.

12. Dra. Berenice Aguirre-Gómez, Dr. José Asz-Sigall, Dr. Francisco Antonio Medina-Vega, Dra. Karla Alejandra Santos-Jasso. Obstrucción pilórica inusual en pediatría. Informe de seis casos operados. Acta Pediatr Mex 2013; 34(1):28-32. 